Utah State University

DigitalCommons@USU

Mechanical and Aerospace Engineering Student Mechanical and Aerospace Engineering Student Publications and Presentations

$1-5-2020$

\title{
Identifying Optimal Equivalent Area Changes to Reduce Sonic Boom Loudness
}

Troy Abraham

Utah State University, troy.abraham@aggiemail.usu.edu

Douglas F. Hunsaker

Utah State University, doug.hunsaker@usu.edu

Jonathan M. Weaver-Rosen

Texas A\&M University

Richard J. Malak Jr.

Texas A\&M University

Follow this and additional works at: https://digitalcommons.usu.edu/mae_stures

Part of the Mechanical Engineering Commons

\section{Recommended Citation}

Abraham, T. A., Hunsaker, D. F., Weaver-Rosen, J. M., and Malak, R. J., "Identifying Optimal Equivalent Area Changes to Reduce Sonic Boom Loudness," AIAA Scitech Forum, Orlando, Florida, January 2020,

AIAA-2020-0790, DOI: 10.2514/6.2020-0790

This Article is brought to you for free and open access by the Mechanical and Aerospace Engineering Student Research at DigitalCommons@USU. It has been accepted for inclusion in Mechanical and Aerospace Engineering Student Publications and Presentations by an authorized administrator of DigitalCommons@USU. For more information, please contact digitalcommons@usu.edu.

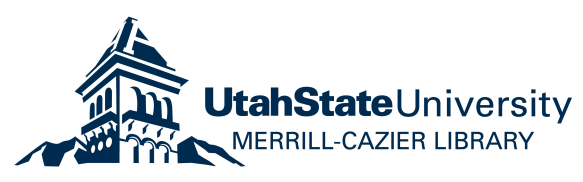




\title{
Identifying Optimal Equivalent Area Changes to Reduce Sonic Boom Loudness
}

\author{
Troy A. Abraham* and Douglas F. Hunsaker. ${ }^{\dagger}$ \\ Utah State University, Logan, UT, 84322 \\ Jonathan M. Weaver-Rosen ${ }^{\ddagger}$ and Richard J. Malak Jr. ${ }^{\S}$ \\ Texas A\&M University, College Station, TX, 77843
}

\begin{abstract}
This work explores the design space created from modeling the effect of localized geometric changes on a supersonic aircraft's near-field pressure signature. These geometric changes are used to alter the aircraft's near-field pressure signature in a way that reduces its sonic boom loudness at the ground. The aircraft used in this work is the NASA 25D concept and its near-field pressure signature is modeled using two seperate methods. The first method uses the PANAIR panel code to obtain a near-field pressure signature for an axisymmetric representation of the 25D. This near-field signature is propagated to the ground using the NASA SBOOM propagation code and the perceived level in decibels is calculated using an in-house loudness code called PyLdB. The second method uses the equivalent area distribution of the 25D which is passed directly to $\mathrm{SBOOM}$ and the perceived level is again found using PyLdB. To model the geometric changes, the axisymmetric geometry and the equivalent area distributions are independently modified with a parameterized Gaussian deformation. These methods are fast enough to quickly explore the design space and find the change in loudness for different deformation parameters. This design space exploration is used to study loudness changes for both on-design conditions and the effects of deviations from on-design angle of attack, Mach number, and azimuth angle. A genetic algorithm is used in subsequent studies to explore the effects of different atmospheric conditions. These results can be used to inform higher fidelity CFD studies and structural adaptation design on the aircraft.
\end{abstract}

\section{Introduction}

The sonic booms produced by civil supersonic aircraft have been a subject of study since the early 1960s. Efforts to bring supersonic aircraft into commercial use have been limited due to the annoyance of the sonic boom loudness to human populations as well as the sonic boom's potential for causing structural damage [1]. Because of these side effects, supersonic flight over the United States is currently restricted and commercial flights in the past have been limited to over-water operation routes [2]. In an effort to overcome the current restrictions to supersonic flight, a number of studies have been performed that explore modifying an aircraft's geometry to change its pressure signature and reduce its sonic boom loudness to a level permissible for overland flight [1].

The current work is part of a NASA University Leadership Initiative titled "Adaptive Aerostructures for Revolutionary Civil Supersonic Transportation", hereafter referred to as the ULI. The ULI is studying the feasibility of using localized aircraft outer mold line (OML) structural adaptivity to reduce an aircraft's sonic boom loudness at off-design flight conditions. Identifying and implementing the required OML changes to return an aircraft's loudness to the required levels for overland flight would expand the range of its realizable operating conditions.

The studies specific to the current work utilize low fidelity methods to explore the design space created from the localized geometric changes to the aircraft's OML. Two low fidelity methods are used in this work and are described in detail in the following section. The NASA 25D concept aircraft and its axisymmetric representation, as provided by the 2017 2nd AIAA Sonic Boom Prediction Workshop, are the aircraft geometries that will be studied in this work [3].

The design space exploration begins with identifying OML changes that reduce loudness in a standard atmospheric profile. These results are used to constrain a genetic algorithm implemented to find optimum changes to the OML for a

\footnotetext{
*Graduate Research Assistant, Mechanical and Aerospace Engineering Dept., 4130 Old Main Hill, AIAA Member.

$\dagger$ Assistant Professor, Mechanical and Aerospace Engineering Dept., 4130 Old Main Hill, AIAA Senior Member.

$\doteqdot$ Graduate Research Assistant, Department of Mechanical Engineering, 3123 TAMU, AIAA Member.

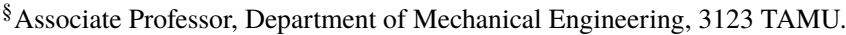


number of different atmospheric conditions. This optimization is performed using both a single geometry change as well as multiple changes at different locations on the aircraft. As a final study, the design space exploration is used to examine off-design conditions in Mach number, angle of attack, and a range of azimuth angles. Comparisons between the unpowered and powered models of the 25D are also made. The results of this work are used to identify the locations and magnitudes of geometric changes that can be used in higher fidelity studies and by a design team developing an adaptive structure capable of morphing in-flight.

\section{Methods}

Two sets of tools and methods are used in the exploration of the design space. The first method produces a near-field pressure signature using the PANAIR panel code and an axisymmetric geometry representation of the aircraft's equivalent area [3, 4]. The equivalent area distribution is the distribution of area that would produce the same near-field pressure signature as the full aircraft geometry [5]. Previous work has demonstrated that PANAIR can be used to produce good near-field results for the 25D's axisymmetric geometry [6].The near-field signature found using PANAIR is passed to the NASA sBOOM Sonic Boom Propagation code to produce a ground signature [7]. The ground signature produced by sBOOM is analyzed using an in-house code called PyLdB to estimate the sonic boom loudness at the ground. This code is based on Stevens' Mark VII method for calculating the perceived level in decibels (PLdB) [8, 9 ${ }^{*}$

In the second method, the aircraft's equivalent area distribution is passed directly to sBOOM. This removes the need of PANAIR for producing the near-field pressure signature. sBOOM propagates the resulting pressure signature to the ground and the loudness is calculate using PyLdB. Each of these methods is described in detail in the sections that follow.

\section{A. Axisymmetric Geometry and PANAIR Panel Code}

The axisymmetric geometry method, hereafter referred to as AXIE, is used to explore the design space of small geometric changes to the OML of the 25D and their effect on PLdB at the ground. The geometry used was provided by the 2017 2nd AIAA Sonic Boom Workshop and the PANAIR panel solution using this geometry was studied extensively in previous work by Giblette [6]. The small geometry changes are achieved by modifying the radius of the AXIE PANAIR mesh with a parameterized Gaussian function to add a single deformation at some axial location. The Gaussian deformation is specified with a length equivalent to one standard deviation, a height or amplitude, and the axial location on the geometry, as shown in Fig. 1. The axisymmetric property of the geometry is conserved by adding the deformation to the entire circumference of the modified section. Figure 2 shows an example of the AXIE PANAIR mesh with a deformation added.

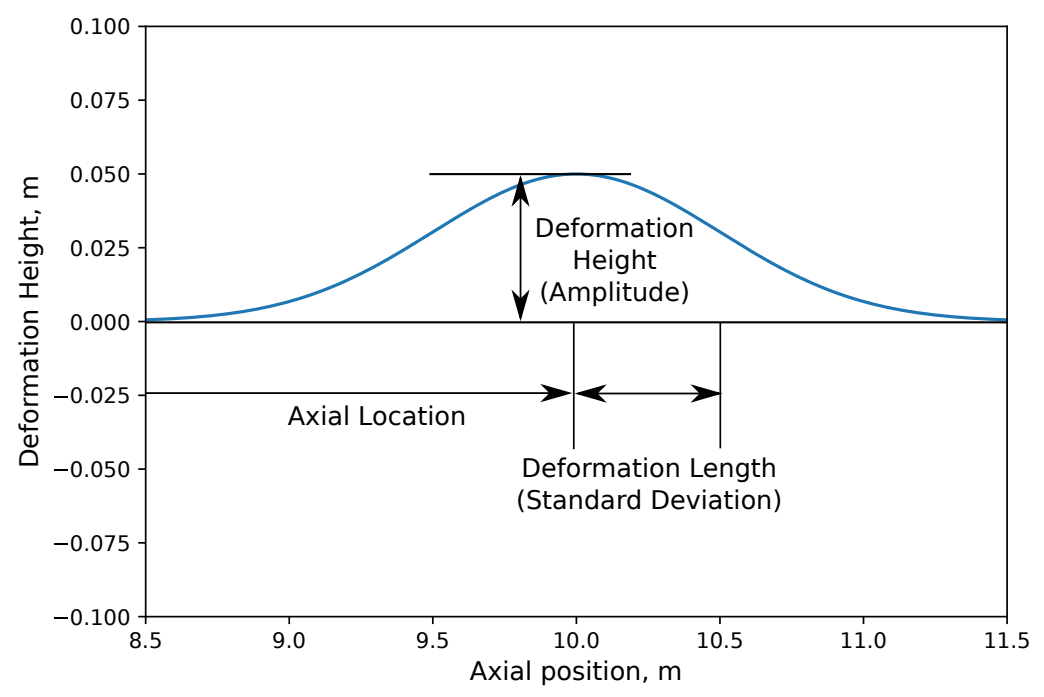

Fig. 1 Gaussian deformation description.

*https://github.com/usuaero/PyLdB 


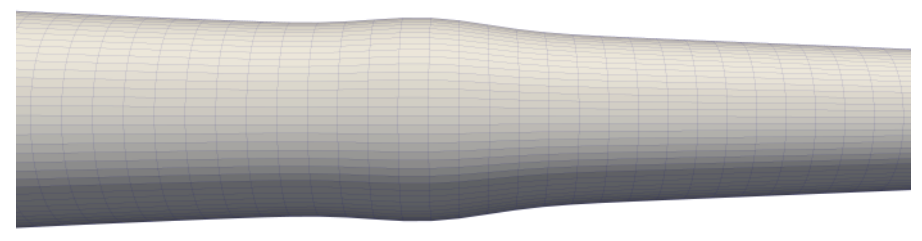

Fig. 2 AXIE geometry with positive deformation.

The design space is explored by analyzing all combinations of deformation parameters within a specified range. A range of positive and negative deformation heights as well as the axial locations are generated with a specified number of evenly spaced values. The full combination of heights and locations are analyzed for a set deformation length and the resulting near-field signatures are propagated to the ground where the loudness of each is recorded. This analysis is performed for multiple deformation lengths until the design space has been explored to a desired level. To visualize the design space, the change in PLdB from the baseline AXIE PLdB for each deformation is mapped on a colored contour plot. These contour plots serve as a simple way to identify the locations and magnitudes of geometry changes that provide a reduction in PLdB.

\section{B. Equivalent Area Distribution}

The near-field pressure disturbances in linearized supersonic flow over a lifting body are affected by lift and cross-sectional area [5]. The contribution of the body's cross-sectional area is found by taking cuts along the Mach plane at locations down the body's axis. The contribution from the lift is calculated by integrating the pressure around the perimeter of the cross-section at each cut. Linearized supersonic flow theory defines a relationship between the near-field pressure disturbance of an aircraft and what is known as the Whitham F-function [10]. The F-function is dependent on the distribution of aircraft area cut by the Mach planes as described above. This method of calculating an equivalent area distribution does not account for the non-linear or higher order flow effects [11]. Because of this, other methods of generating an equivalent area distribution are more desirable.

$\mathrm{Li}$ and Rallabhandi [11] present an equation that demonstrates the relationship between the F-function and the near-field pressure disturbance $(\mathrm{dp} / \mathrm{p})$ and provides a way to calculate a reversed equivalent area distribution from a near-field pressure signature. This approach effectively captures the non-linear effects of the flow [11]. In this work, the near-field signature for the NASA 25D is found for different flight conditions using a full Euler CFD solution. Additional near-field signatures from the 2017 2nd AIAA Sonic Boom Prediction Workshop are also used. These near-field signatures are converted to equivalent area distributions using a code based on the equation outlined in Li and Rallabhandi [11] This method provides a way to more closely couple results from the high and low fidelity methods being used in the ULI.

The equivalent area method is implemented in a manner similar to the AXIE method in exploring the design space. The equivalent area distribution is modified with a similar parameterized Gaussian deformation. The height of the deformation represents the amplitude of the change in area and the length of the deformation is the range over which the change influences the area distribution. Figure 3 shows the variation in the equivalent area achieved by adding a Gaussian deformation to the original distribution. The design space is explored in the same way as the AXIE, with each study determined by a deformation length with varying deformation height and location. Contour plots are generated for the difference in PLdB from the baseline for comparison.

\section{Off Design Flight Conditions}

In an effort to identify more robust aircraft geometry changes for reducing sonic boom loudness, the design space is explored for a number of different off-design flight conditions. The aircraft flight variables considered in this study are perturbations in Mach number, angle of attack, and azimuth angle. Atmospheric profiles with varying temperature, humidity, wind, and effective height above ground are explored as well. Visual representations of the design space, as described previously, are used to identify locations and magnitudes of geometric aircraft changes most effective at reducing loudness.

\footnotetext{
†https://github.com/usuaero/rapidboom
} 


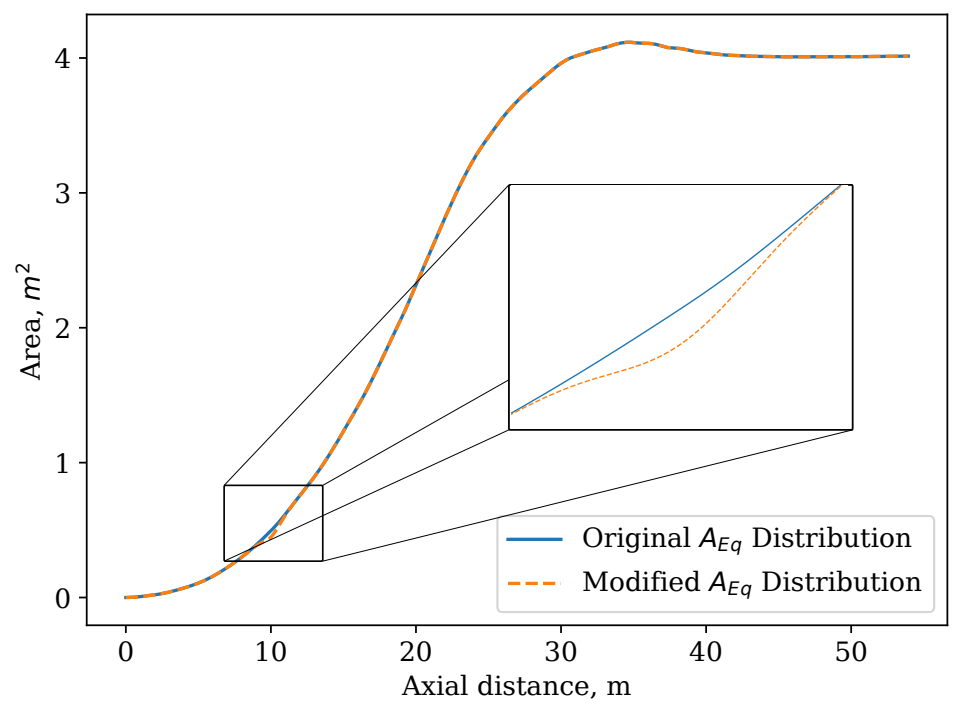

Fig. 3 Modified and unmodified equivalent area distributions.

The use of the equivalent area method provides a significant reduction in computation time. Because of this, the equivalent area method is used for the bulk of the design space exploration. Specifically, the results for the standard atmospheric design condition are obtained using both the PANAIR AXIE and equivalent area methods for comparison, while all off-design flight studies are done using the equivalent area method alone.

\section{Standard Atmosphere: PANAIR and Equivalent Area Results}

This work begins with exploring the effects of geometric changes to a supersonic aircraft in a standard atmospheric profile with no wind. The standard atmospheric profile is built into the NASA sBOOM code and defines a relative humidity for a given altitude [7]. The following studies are done using a cruise altitude of 45,000 ft $(13.716 \mathrm{~km})$. The results using the standard atmospheric profile provide a baseline for comparison in the proceeding studies.

\section{A. AXIE Geometry Design Space}

The AXIE design space is explored and visualized by analyzing cases for combinations of Gaussian deformations over a range of heights, lengths, and locations. The range of axial locations includes 140 values from 0 to $50 \mathrm{~m}$ and the range of deformation heights includes 60 values between $\pm 0.05 \mathrm{~m}$. The deformation lengths used are $0.125,0.25,0.5$, $0.75,1.0,1.5$, and $2.0 \mathrm{~m}$. Figure 4 shows the design space using a Gaussian deformation with a length of $1.5 \mathrm{~m}$ where the contours show a change in PLdB from the baseline. Regions of blue indicate a reduction in PLdB and regions of red indicate an increase from the baseline.

Figure 4 shows clear and significant minima at 12 and $35 \mathrm{~m}$ and the deformation heights at these minima indicate that a reduction in the cross-sectional area is necessary to provide a reduction in PLdB. Plots of other deformation lengths show similar results but with varying degrees of PLdB reduction. Another cut through the design space can be visualized by varying deformation height and length while holding the axial location constant. The results shown in Fig. 5 show the minimum at a deformation length between 1.0 and $1.75 \mathrm{~m}$. Figure 5 also shows that loudness is less dependent on deformation length than on height and location.

The minimum for the cases analyzed using the AXIE method was found to be a reduction of 1.73 PLdB for a deformation located at $34.89 \mathrm{~m}$ with a height and length of $-0.0059 \mathrm{~m}$ and $1.5 \mathrm{~m}$ respectively. Figure 6 provides a visual representation of the full design space with the axes of the figure representing the different deformation parameters.

\section{B. Equivalent Area Design Space}

The equivalent area design space is visualized in the same way as the AXIE design space. The major difference between the two methods being that the equivalent area distribution is modified and changed directly using the Gaussian 


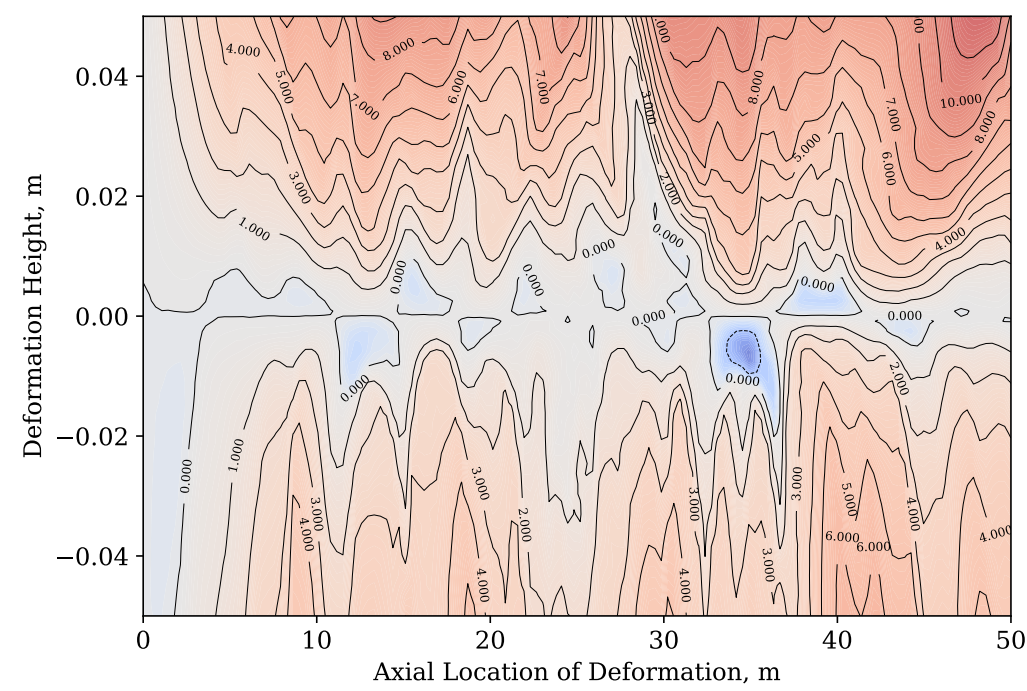

Fig. 4 Change in PLdB from baseline using AXIE model with a deformation length of $1.5 \mathrm{~m}$.

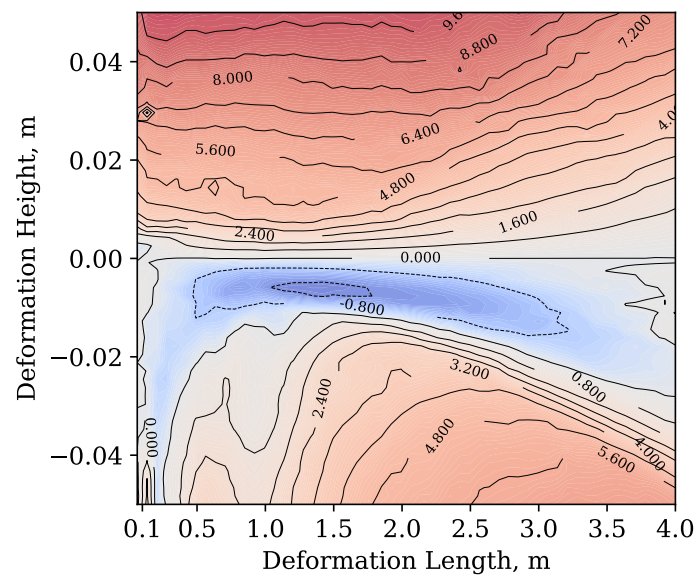

Fig. 5 Change in PLdB from baseline using AXIE model with an axial location of $35 \mathrm{~m}$.

function. The range of the Gaussian amplitudes is changed to $\pm 0.35 \mathrm{~m}^{2}$ because it now represents a change in area rather than radius as in the AXIE method. Figure 7 shows the equivalent area design space with the same $1.5 \mathrm{~m}$ deformation length as the AXIE plot (Fig. 4).

There are similarities between the results of the equivalent area method and those of the AXIE method. There are minima around the 12, 32, and $35 \mathrm{~m}$ locations, just as with the AXIE results. The equivalent area method uses a slightly different approach than the AXIE method to explore this design space but, even though magnitudes of the minima are different, the similarities in locations and the other trends seen in the design space are very similar.

The goal of these studies is to provide locations and magnitudes of localized changes in geometry that are feasible for the actual aircraft geometry. This means that the results need to be constrained to magnitudes and locations that are feasible for the methods and techniques being used to accomplish the physical geometry changes. The design space exploration is useful as a first approach for gaining an understanding of the design space and, using these results, identifying realizable locations and magnitudes of geometry changes.

Figure 8 shows an equivalent area design space for a Gaussian deformation with a $1 \mathrm{~m}$ standard deviation. An image of the NASA 25D with Mach lines is included for reference at the top of the figure. The minimum around the $12 \mathrm{~m}$ mark appears to correspond to the area cut through the fuselage just before the wing. The minima at the $32 \mathrm{~m}$ mark 


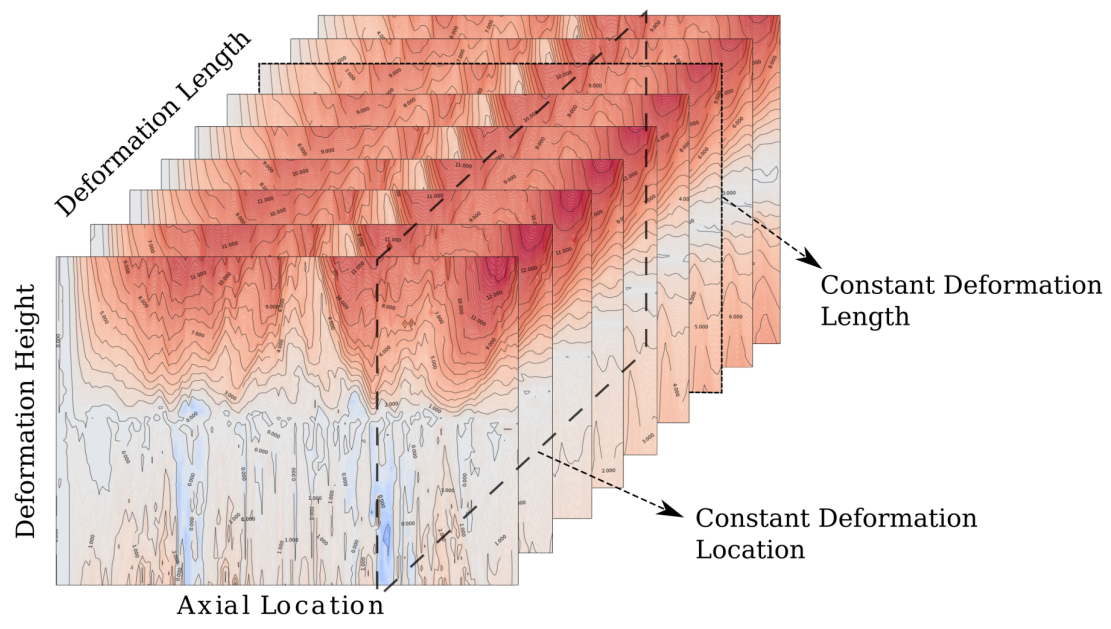

Fig. 6 Complete AXIE deformation design space.

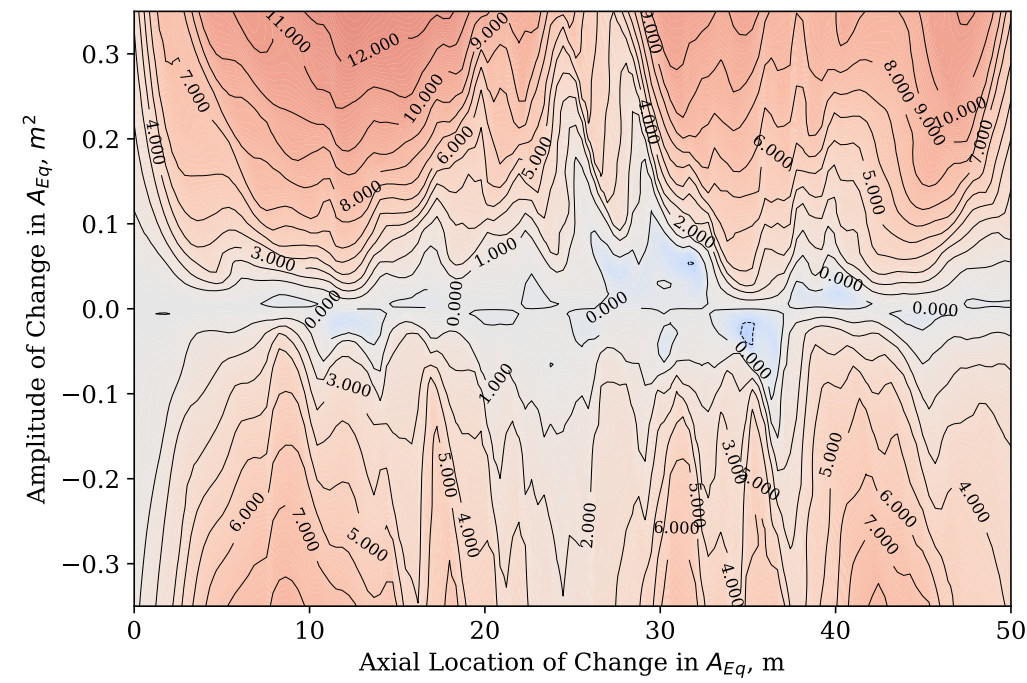

Fig. 7 Change in PLdB from baseline using the equivalent area model with a deformation length of $1.5 \mathrm{~m}$.

correspond to the areas around the engine and the vertical and horizontal stabilizers. Locations further back than the 33 $\mathrm{m}$ mark correspond to area contributions from the vertical stabilizer and aircraft wake, which makes them difficult, if not impossible, to modify. Because of this, constraints used in the optimization studies are set to hold deformations to an axial location within $32.92 \mathrm{~m}$ (length of 25D). Deformation heights and lengths are also constrained to magnitudes between $\pm 0.05 \mathrm{~m}$ and $0.125 \mathrm{~m}$ to $2 \mathrm{~m}$ respectively. These constraints were chosen as rough estimates of the ranges that would be considered feasible for adaptive aircraft structure design.

\section{Optimization Benchmarking}

The optimization routine used in this paper is the predictive parametric Pareto genetic algorithm (P3GA) [12]. It should be noted that the parametric part of the algorithm is not utilized in this study, and the algorithm therefore simplifies to a method similar to NSGA-II [13]. While this problem does not include changing parameters by P3GA's definition, later studies will include such parameters, giving P3GA an advantage over other methods. Additionally, the multimodal nature of the design space, as seen by the initial design space exploration, is well-suited for genetic algorithms such as P3GA since they are more likely to find a global solution compared to gradient methods. 


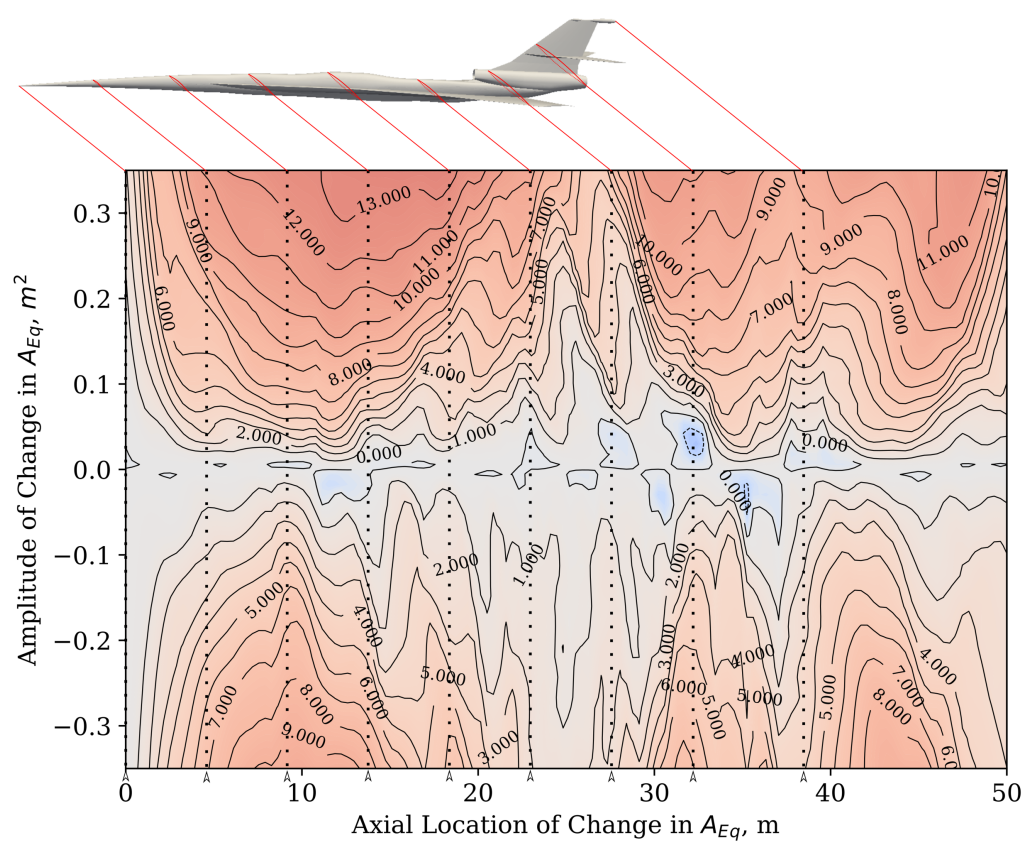

Fig. 8 Change in PLdB from baseline using equivalent area model with physical representation of 25D aircraft for reference.

To benchmark P3GA on this design space, the optimizer and AXIE method are implemented together with the objective of minimizing the loudness with respect to the three aforementioned design variables: location, height, and length of a deformation. The constraints of the problem are the same bounds used in the initial AXIE design space exploration. The solution is a deformation located at $35.04 \mathrm{~m}$ along the axis with a height and length of $-0.0065 \mathrm{~m}$ and $1.30 \mathrm{~m}$, respectively. This falls within the optimal basin seen in Fig. 4 and 5 for the AXIE design space exploration.

\section{Historical Atmospheric Data: Optimization via Equivalent Area}

The following studies use the equivalent area method with a Gaussian area change defined by three design variables for the location and geometry of the deformation. Constraints identified in the equivalent area study are implemented to bound the design space and keep the Gaussian definition accurate. The problem can be stated mathematically as:

$$
\Delta P L d B^{*}=\left[\min _{\mathbf{x}} F(\mathbf{x})\right]-P L d B_{0}
$$

Subject to:

$$
\begin{gathered}
g(\mathbf{x})=\left|\frac{\text { amplitude }}{\text { length }}\right| \leq 0.02 \\
{\left[\begin{array}{c}
0 \\
-0.05 \\
0.125
\end{array}\right] \leq\left[\begin{array}{c}
\text { location } \\
\text { amplitude } \\
\text { length }
\end{array}\right] \leq\left[\begin{array}{c}
32.92 \\
0.05 \\
2
\end{array}\right]}
\end{gathered}
$$

where $\triangle P L d B^{*}$ represents the optimal change in perceived loudness, $P L d B_{0}$ denotes the perceived loudness for the undeformed model, and the design variable vector $\mathbf{x}$ contains the location, amplitude, and length variables for each deformation. $F(\mathbf{x})$ represents the model that uses the design variables to calculate loudness via the equivalent area method. For every geographic location chosen, and its corresponding weather data, the optimal change in perceived loudness is solved using Eq.(1). The weather data used in this study is comprised of weather measurements taken on June 18, 2018 gathered by the National Oceanic and Atmospheric Administration (NOAA) and is passed as input to the

\footnotetext{
$\stackrel{+}{+}$ This data is directly pulled from twisterdata.com, a website developed and maintained by David Demko and Donald Giuliano.
} 
NASA sBOOM code.

When a single deformation is allowed, results show all deformations located in either the 11-12 $\mathrm{m}$ range or the 32-33 m range (see Table 1). Recall that these two locations were identified as local minima from the design space exploration of the standard atmosphere. Additionally, the results show that area should be removed in the 11-12 m range and added in the 32-33 m range to reduce the perceived loudness on the ground for all historical weather conditions examined thus far.

Table 1 Optimal single deformation for multiple atmospheres.

\begin{tabular}{cccccc}
\hline & & \multicolumn{4}{c}{ Deformation } \\
\cline { 3 - 5 } Latitude $\left(^{\circ}\right)$ & Longitude $\left({ }^{\circ}\right)$ & Location $(\mathrm{m})$ & Amplitude $\left(\mathrm{m}^{2}\right)$ & Length $(\mathrm{m})$ & $\Delta P$ L $d B^{*}(\mathrm{~dB})$ \\
\hline 34 & -118 & 32.38 & 0.0198 & 0.992 & -0.84 \\
35 & -112 & 32.39 & 0.0195 & 0.974 & -1.01 \\
36 & -105 & 11.74 & -0.0121 & 0.744 & -0.82 \\
37 & -99 & 11.78 & -0.0137 & 0.826 & -0.78 \\
38 & -93 & 11.78 & -0.0129 & 0.716 & -0.82 \\
39 & -87 & 32.66 & 0.0153 & 0.766 & -0.84 \\
40 & -80 & 32.35 & 0.0200 & 0.999 & -1.03 \\
\hline
\end{tabular}

Upon further inspection of the single deformation case, local minima are discovered at both the 12 and $32 \mathrm{~m}$ locations for all atmospheric profiles studied. However, one of these deformation locations is not optimal for all atmospheric conditions, as observed by the information in Table 1 . For some atmospheric profiles, the $32 \mathrm{~m}$ location is more optimal than the $12 \mathrm{~m}$ location, where for other profiles the opposite is true. An area of future work is to determine what atmospheric property or combination of atmospheric properties contribute to this phenomenon.

When two deformations are allowed simultaneously, results show that the loudness at these same geographic locations decreases by having one deformation near $12 \mathrm{~m}$ and another near $32 \mathrm{~m}$ (see Table 2). These results coupled with the single deformation results point to these deformation locations being optimal for many reasonable atmospheric conditions. Additionally, the two-deformation results do not yet show diminishing returns for adding a second deformation. In fact, the reduction in sonic boom loudness is very nearly a linear superposition of the two individual deformations. The atmospheric profiles studied so far comprise only a small sample of reasonable profiles. However, these results warrant the exploration of deforming the NASA 25D concept near 12 and 32 meters. These locations will serve as initial areas of interest for deformations applied to the three-dimensional high fidelity model, as shown in Fig. 9

Table 2 Optimal double deformation for multiple atmospheres.

\begin{tabular}{ccccccccc}
\hline & & \multicolumn{3}{c}{ Deformation 1 } & \multicolumn{3}{c}{ Deformation 2 } \\
\cline { 3 - 7 } $\begin{array}{c}\text { Latitude } \\
\left({ }^{\circ}\right)\end{array}$ & $\begin{array}{c}\text { Longitude } \\
\left({ }^{\circ}\right)\end{array}$ & $\begin{array}{c}\text { Location } \\
(\mathrm{m})\end{array}$ & $\begin{array}{c}\text { Amplitude } \\
\left(\mathrm{m}^{2}\right)\end{array}$ & $\begin{array}{c}\text { Length } \\
(\mathrm{m})\end{array}$ & $\begin{array}{c}\text { Location } \\
(\mathrm{m})\end{array}$ & $\begin{array}{c}\text { Amplitude } \\
\left(\mathrm{m}^{2}\right)\end{array}$ & $\begin{array}{c}\text { Length } \\
(\mathrm{m})\end{array}$ & $\begin{array}{c}\Delta P L d B^{*} \\
(\mathrm{~dB})\end{array}$ \\
\hline 34 & -118 & 11.77 & -0.0118 & 0.722 & 32.27 & 0.0207 & 1.035 & -1.88 \\
35 & -112 & 11.74 & -0.0128 & 0.777 & 31.98 & 0.0167 & 1.061 & -1.54 \\
36 & -105 & 11.77 & -0.0098 & 0.723 & 28.37 & 0.0102 & 0.753 & -1.27 \\
37 & -99 & 11.74 & -0.0147 & 0.977 & 32.25 & 0.0204 & 1.021 & -2.17 \\
38 & -93 & 11.82 & -0.0130 & 0.772 & 32.33 & 0.0195 & 0.977 & -2.03 \\
39 & -87 & 11.81 & -0.0131 & 0.733 & 32.27 & 0.0204 & 1.021 & -2.09 \\
40 & -80 & 11.96 & -0.0146 & 1.307 & 32.31 & 0.0168 & 0.887 & -1.78 \\
\hline
\end{tabular}

\section{Off-Design Flight Conditions}

An aircraft capable of changing its sonic boom signature during flight will be most useful if it can do so over a wide range of supersonic flight conditions. This study examines the effects of Mach number, angle of attack, and 


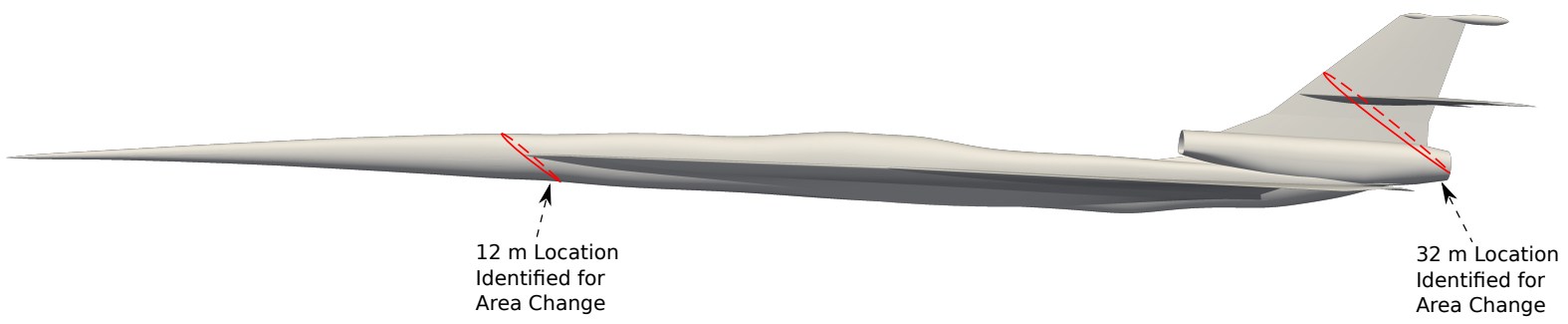

Fig. 9 Mach angle cuts of locations of interest identified on full 25D geometry.

azimuth angle on the optimal deformations for reducing loudness. A brief study is done on how using an equivalent area distribution from a powered CFD model versus an unpowered flow-thru model also affects the optimal deformations. Examining the effects of these conditions using the models previously built for the standard flight conditions allows for easy comparison. Near-field pressure distributions are found using Euler CFD solutions for the 25D at the varying flight conditions listed above. These pressure distributions are converted to equivalent area distributions using the reverse equivalent area code described previously.

In these studies, the equivalent area method is used to explore the design space for each flight condition. This design space is the same as described in the standard atmosphere study where the three dimensions of the design space are the deformation location, height, and length. The PLdB is found for 90000 data points using 100 different axial locations, 30 heights, and 30 deformation lengths. The optimal equivalent area deformation for reducing loudness from the baseline for both the fore and aft locations of the aircraft are identified.

\section{A. Mach Number}

We first consider small deviations in Mach number. The equivalent area distributions for Mach numbers of 1.55, 1.6 (on-design), and 1.65 are generated from full Euler CFD solutions. All other flight parameters are held constant in a standard atmospheric profile at 50,000 ft $(15.24 \mathrm{~km})$. Figure 10 shows the amplitude, length, location, and resulting PLdB for the optimal fore and aft deformations.

The design space exploration results show that both the $12 \mathrm{~m}$ and $32 \mathrm{~m}$ locations remain optimal for each of the Mach numbers studied. The amplitude of the deformations at those locations are found to have very little sensitivity to deviations in Mach number. The length of the deformations show some variation at the lowest Mach number, but are otherwise consistent. Figure 10 also shows that PLdB is fairly sensitive to Mach number over the range studied. At a Mach number of 1.55 the fore location provides a greater reduction in PLdB than the aft location, which is opposite from all previous results. This indicates there is variation in the effectiveness of these deformations with respect to deviations in Mach number.

These results indicate that implementing structural adaptivity to change the equivalent area at both of these locations, 12 and $32 \mathrm{~m}$, remains a viable approach to reducing loudness, even with changing Mach number. The change in optimal location for Mach 1.55 indicates some variation exists in this range of Mach numbers and future work should focus on studying a larger range of Mach numbers with more intermediate Mach values.

\section{B. Angle of Attack}

The aircraft's angle of attack is the next off-design parameter studied. The equivalent area distributions for angles of attack of 2.975, 3.375 (on-design), and 3.575 degrees are generated from full Euler CFD solutions. Figure 11 shows the resulting optimal fore and aft deformation parameters and loudness.

The design space exploration results are similar to those seen in the Mach number and historical atmospheric studies. The amplitude and length of the aft deformations are far more sensitive to angle of attack than the the amplitude and length of the fore deformations. The optimal deformation locations appear to be insensitive to angle of attack. These results demonstrate that the optimal deformations have some dependence on angle of attack, but do not vary significantly from the on-design results.

Figure 11 also shows that the achievable loudness is sensitive to angle of attack. The aft location is optimal for both the on-design and smaller angles of attack but the optimal switches to the fore location for the larger angle of attack, similar to what was seen for a smaller Mach number. These results continue to support the trend of a bimodal 

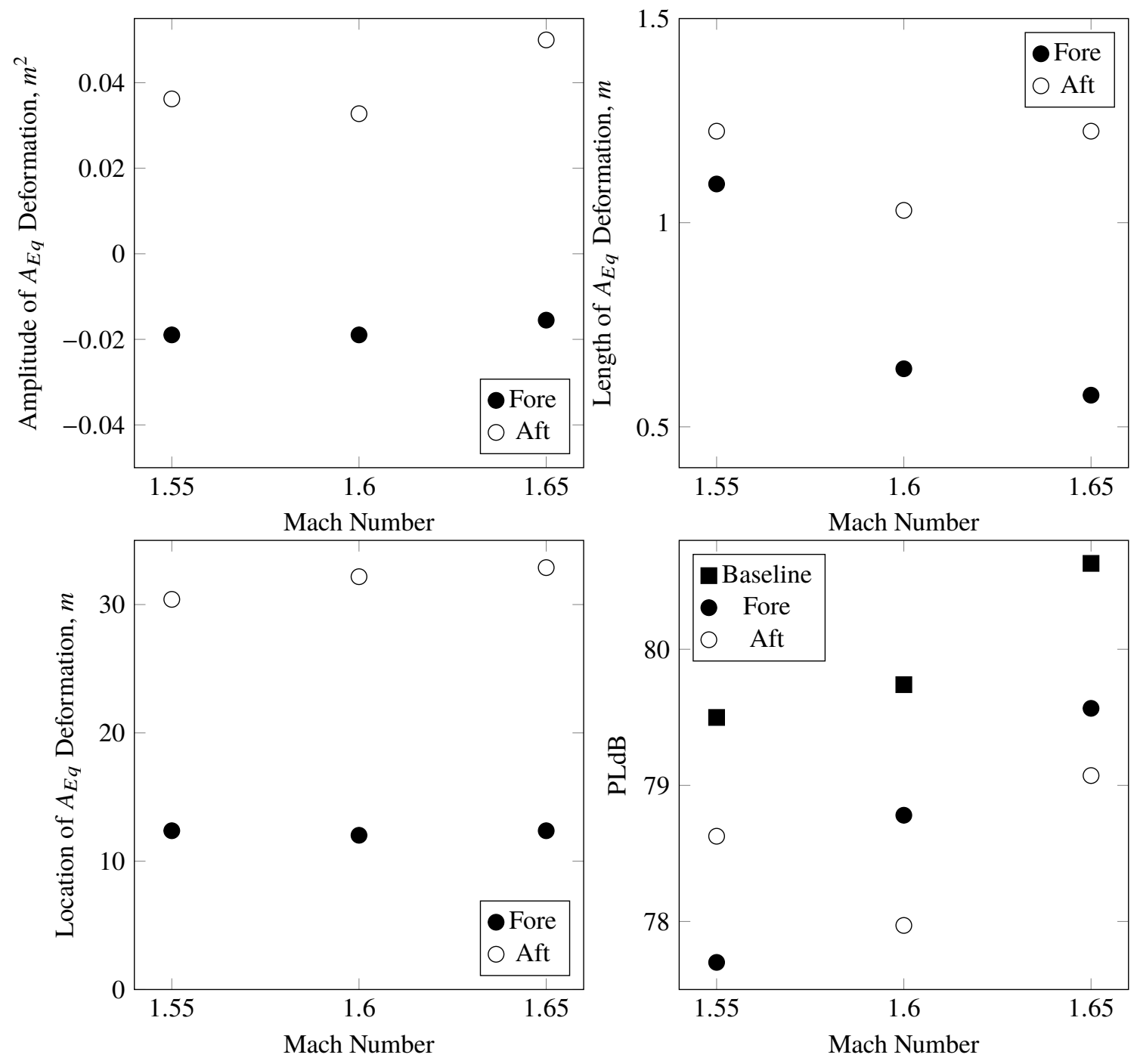

Fig. 10 Optimal deformation results vs. Mach number. 

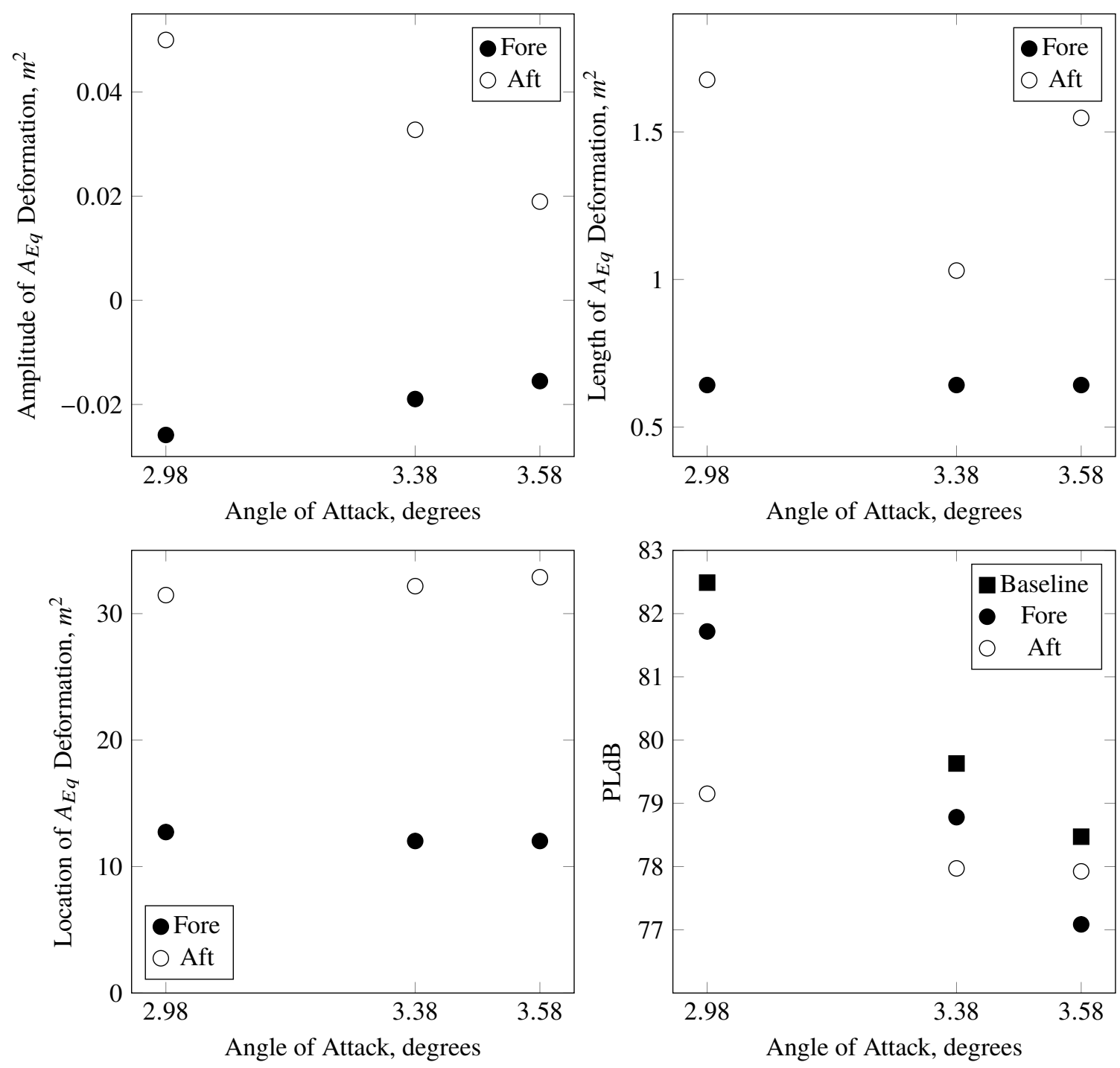

Fig. 11 Optimal deformation results vs. angle of attack. 
optimum that demonstrates the fore and aft locations being necessary to recover from the increase in PLdB caused by these off-design flight parameters. Again, future work should focus on a larger and more populated range of angle of attack, which could provide more insight to the variations seen in the optimal deformation across the current range of angles of attack.

\section{Azimuth Angle}

Sensitivity to azimuth angle was also considered. The near-field signatures from Euler solutions for azimuth angles between 0 and 50 degrees at 2 degree increments are converted to equivalent area distributions. Figure 12 shows the azimuth angle as defined from the undertrack of the aircraft.

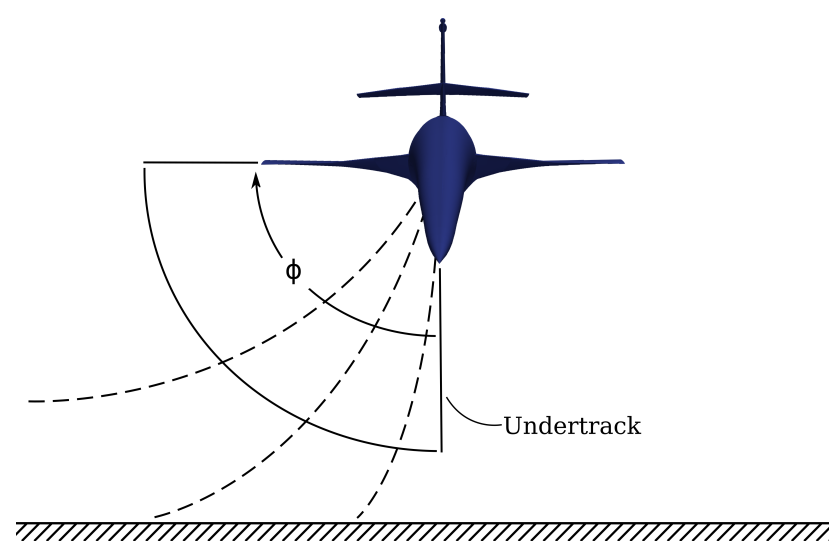

Fig. 12 Azimuth angle measured from undertrack of the aircraft.

The optimal fore and aft deformations are identified and recorded using the same design space exploration methods described previously. Figure 13 shows the optimal deformation parameters as functions of azimuth angle for the fore and aft locations of the aircraft.

Figure 13 shows that the magnitudes and locations of the optimal deformation amplitudes are consistent with those seen in previous studies and are fairly insensitive to azimuth angle. The length of these optimal deformations shows a large sensitivity to azimuth angle; although, we have seen that, for a standard atmosphere, the loudness produced is less sensitive to deformation length than the other parameters.

Figure 13 also shows large sensitivity to azimuth angles in the baseline and optimal PLdB produced using a fore and aft deformation. The maximum PLdB occurs from the near-field signature at an azimuth angle around 30 degrees and an aft deformation always provides a larger reduction in PLdB from the baseline. The results in Figure 13 show consistency in the optimal location and amplitude of deformations but significant changes in length are necessary to meet that optimum for different azimuth angles.

\section{Powered Model}

One model that the Sonic Boom Workshop has additional results for is a powered aircraft with the engine plume. An equivalent area model is created from the Workshop's near-field pressure signature of this powered aircraft and implemented in the design space exploration. The powered pressure signature is only available for on-design flight conditions. Therefore, the only comparisons that are made are to the unpowered on-design results.

Figure 14 shows loudness contours for the unpowered and powered models. A comparison of these plots shows some variations in the PLdB minima. The minimum at the fore location appears mostly unchanged while the aft minimum has shifted further back from the $32 \mathrm{~m}$ location. The baseline PLdB for the powered model is $81.10 \mathrm{PLdB}$, or about 1.5 PLdB greater than the unpowered model. The aft deformation on the powered model is able to produce a 1.58 decrease in PLdB from the baseline, down to 79.52 PLdB, while the aft deformation on the unpowered model produces a 1.66 decrease in PLdB, down to 77.97 PLdB.

Ideally, future work would incorporate more use of the powered model at the different flight conditions examined thus far. The results of the baseline powered and unpowered model comparison show the aft deformation shifting further 

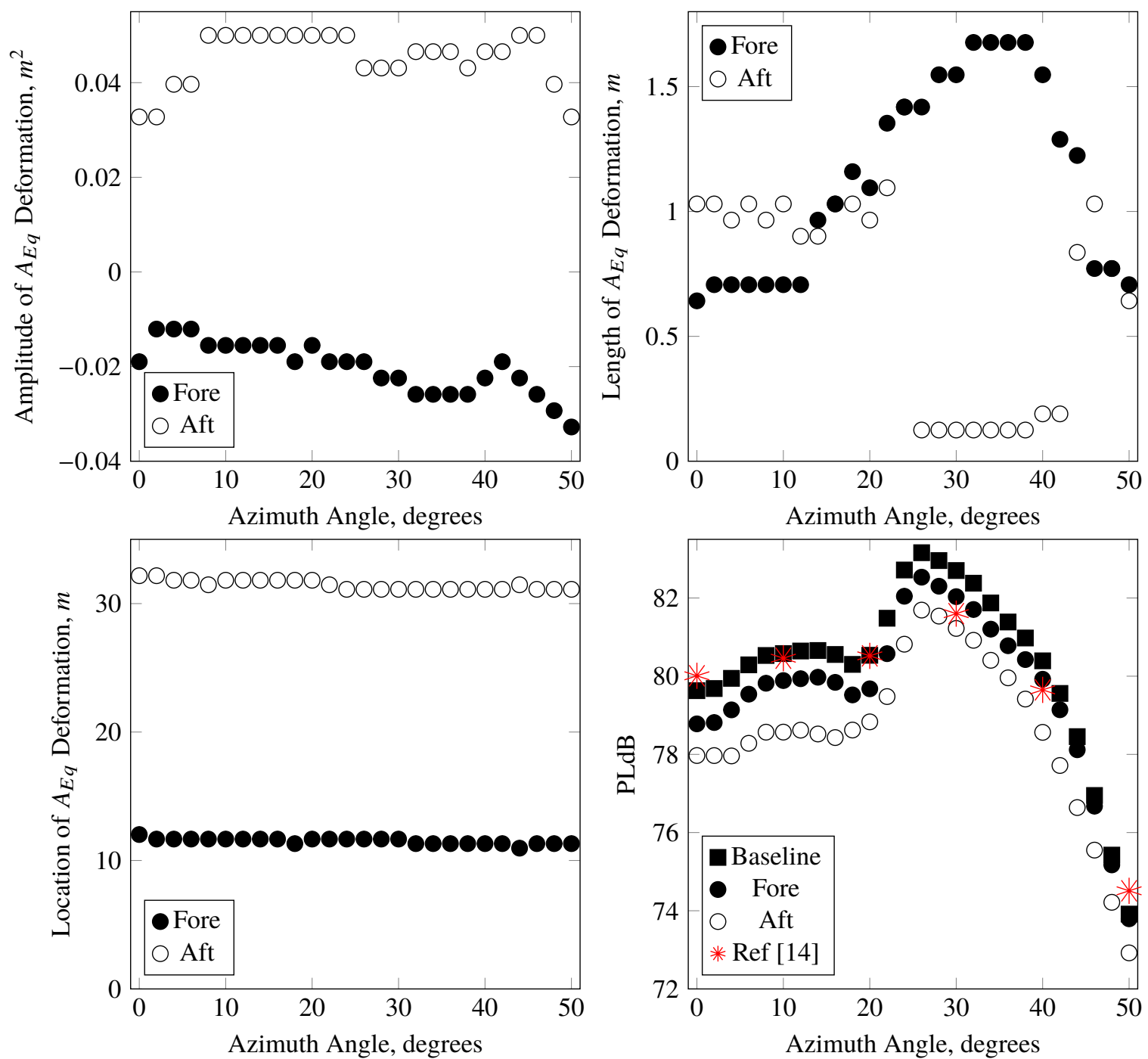

Fig. 13 Optimal deformation results vs. azimuth angle.
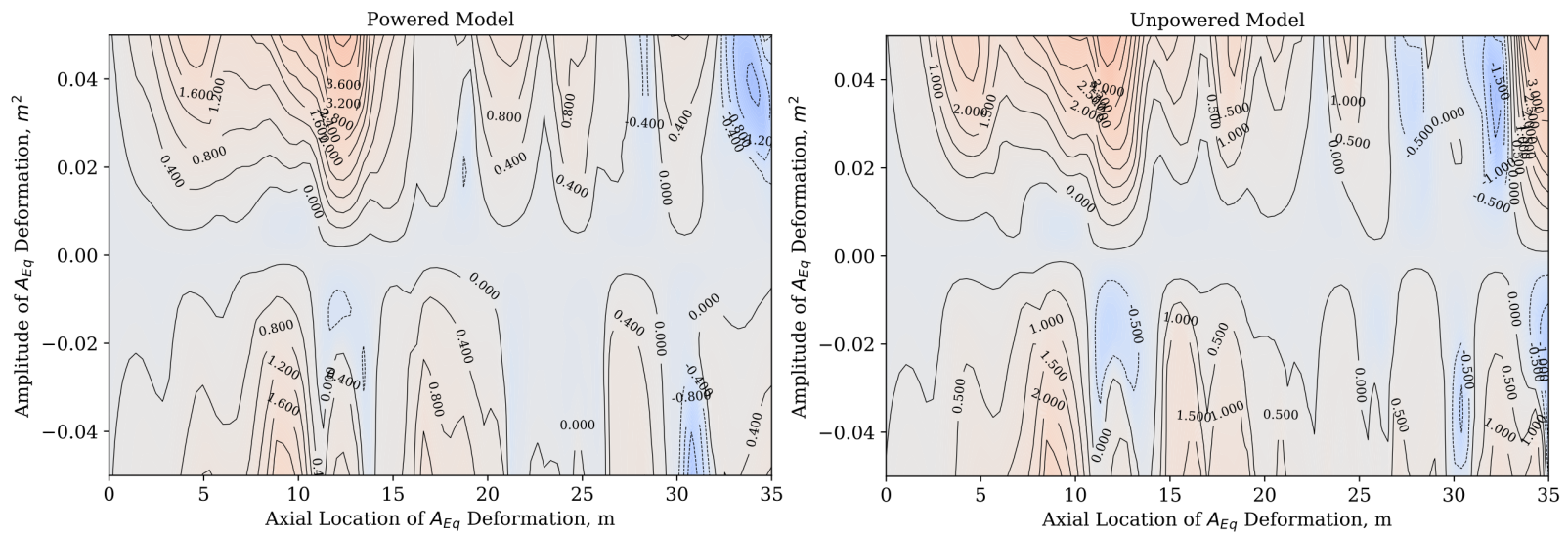

Fig. 14 Change in PLdB from baseline using equivalent area model for unpowered and powered 25D with deformation lengths of 1.03 and $1.16 \mathrm{~m}$ respectively. 
back on the aircraft, to somewhere on the vertical stabilizer. These results are still useful for informing the higher fidelity studies with locations of interest to deform the full three-dimensional geometries.

\section{Conclusion}

A low fidelity equivalent area method was used to explore the design space of localized geometric changes to the NASA 25D's OML. The geometric changes are used to alter the aircraft's near-field pressure signature in a way that reduces its sonic boom's loudness at the ground. The equivalent area method design space is shown to have the same minima as an alternative design space that was explored using the PANAIR panel code and axisymmetric geometry. The design space exploration was used to identify locations on the aircraft's geometry that can be modified to minimize PLdB. Locations at 12 and $32 \mathrm{~m}$ on the NASA 25D concept aircraft show the most promise for reducing loudness.

Optimal locations for area change in varying atmospheric profiles were found using a genetic algorithm and the equivalent area method. Minima were consistently found at the 12 and $32 \mathrm{~m}$ locations and reducing the loudness on the undertrack was possible with a maximum change of -2.17 PLdB seen in the two deformation case.

The equivalent area design space exploration was used to study the effects of small perturbations to on-design flight conditions. The optimal deformations for changes in angle of attack, Mach number, and azimuth angle were found. The off-design results show the locations and magnitudes of the deformations remain consistent. There is some variation in which deformation produces the greatest PLdB reduction, but the minimums remain at the $12 \mathrm{~m}$ and $32 \mathrm{~m}$ locations. The use of a powered aircraft model was shown to shift the aft minimum slightly further back.

While the equivalent area representation is not a one-to-one mapping back to a three-dimensional aircraft geometry, it can be used to narrow down the design space for higher fidelity CFD studies in the ULI. These results can also be used to inform preliminary design steps for the ULI team designing the adaptive aircraft structures.

\section{Acknowledgments}

This work is supported by the NASA University Leadership Initiative (ULI) program under federal award number NNX17AJ96A, titled "Adaptive Aerostructures for Revolutionary Civil Supersonic Transportation."

\section{References}

[1] Maglieri, D. J., Bobbitt, P. J., Plotkin, K. J., Shepherd, K. P., Coen, P. G., , and Richwine, D. M., "Sonic Boom: Six Decades of Research,” Technical Report NASA/SP-2014-622, NASA, 2014.

[2] Federal Aviation Regulations, “14 C.F.R. §91.817, "Civil aircraft sonic boom",”, 2019.

[3] Park, M. A., and Nemec, M., "Nearfield Summary and Statistical Analysis of the Second AIAA Sonic Boom Prediction Workshop," 35th AIAA Applied Aerodynamics Conference, 2017.

[4] Derbyshire, T., and Sidwell, K. W., "PAN AIR Summary Document," NASA Contractor Report, Boeing Military Airplane Company, Seattle, WA, 1982.

[5] Seebass, A. R., “Sonic Boom Theory,” Journal of Aircraft, Vol. 6, No. 3, 1969, pp. 177-184.

[6] Giblette, T., and Hunsaker, D. F., "Prediction of Sonic Boom Loudness Using High-Order Panel Methods for the Near-Field Solution," AIAA Scitech 2019, San Diego, 2019.

[7] Rallabhandi, S. K., "Advanced Sonic Boom Prediction Using the Augmented Burgers Equation,” Journal of Aircraft, Vol. 48, No. 4, 2011, pp. 1245-1253.

[8] Bolander, C. R., Hunsaker, D. F., Shen, H., and Carpenter, F. L., "Procedure for the Calculation of the Perceived Loudness of Sonic Booms," AIAA Scitech 2019, San Diego, 2019.

[9] Stevens, S., "Perceived level of noise by Mark VII and decibels (E)," The Journal of the Acoustical Society of America, Vol. 51, No. 2B, 1972, pp. 575-601.

[10] Plotkin, K. J., "Review of Sonic boom Theory," AIAA 12th Aeroacoustics Conference, 1989.

[11] Li, W., and Rallabhandi, S. K., "Inverse Design of Low-Boom Supersonic Concepts Using Reversed Equivalent-Area Targets," Journal of Aircraft, Vol. 51, No. 1, 2014, pp. 29-36. 
[12] Galvan, E., and Malak, R. J., "P3GA: An Algorithm for Technology Characterization,” Journal of Mechanical Design, Vol. 137, 2015.

[13] Deb, K., Pratap, A., Agarwal, S., and Meyarivan, T., "A fast and elitist multiobjective genetic algorithm: NSGA-II," IEEE Transactions on Evolutionary Computation, Vol. 6, 2002, pp. 182-197.

[14] Ishikawa, H., Makino, Y., Ueno, A., and Kanamori, M., "Sonic Boom Assessment in Primary Boom Carpet of Low-Boom Supersonic Airplane (NASA C25D),” AIAA Scitech 2019, San Diego, 2019. 